Research Article

\title{
Games under Ambiguous Payoffs and Optimistic Attitudes
}

\author{
Wei Xiong \\ Department of Philosophy, Institute of Logic and Cognition, Sun Yat-Sen University, Guangzhou 510275, China \\ Correspondence should be addressed to Wei Xiong; hssxwei@mail.sysu.edu.cn
}

Received 28 August 2013; Revised 24 December 2013; Accepted 24 December 2013; Published 6 February 2014

Academic Editor: Guiomar Martín-Herrán

Copyright (C) 2014 Wei Xiong. This is an open access article distributed under the Creative Commons Attribution License, which permits unrestricted use, distribution, and reproduction in any medium, provided the original work is properly cited.

\begin{abstract}
In real-life games, the consequence or payoff of a strategy profile and a player's belief about the consequence of a strategy profile are often ambiguous, and players may have different optimistic attitudes with respect to a strategy profile. To handle this problem, this paper proposes a decision rule using the Hurwicz criterion and Dempster-Shafer theory. Based on this rule, we introduce a new kind of games, called ambiguous games, and propose a solution concept that is appropriate for this sort of games. Moreover, we also study how the beliefs regarding possible payoffs and optimistic attitudes may affect the solutions of such a game. To illustrate our model, we provide an analysis of a scenario concerning allocating resource of defending and attacking in military contexts.
\end{abstract}

\section{Introduction}

Game theory is a powerful tool for analyzing strategic interactions between decision makers in many domains, such as voting, economics, and artificial intelligence. In this theory, a strategic (also called normal form or static) game is used to model a strategic situation, in which it is assumed that the consequence or payoff of a pure strategy profile is determinate or precise. According to this model, many kinds of games and their corresponding solutions can be defined.

In real-life games, the players have to make decisions under ambiguity because there is often only limited information available to the decision maker. In canonical opinion, ambiguity is referred to as a kind of uncertainty that describes situations where decision makers cannot determine a precise probability distribution over the possible consequences of an action [1-3]. Therefore, in games under ambiguity, the players could only assign a set of possible payoffs, rather than a precise payoff, to a pure strategy profile and have an imprecise probability distribution over this set. Moreover, the players often have some optimistic attitudes (decision attitudes), including optimistic, neutral, and pessimistic, in the decision problems under ambiguity [4-7]. In view of this, this aforementioned assumption of strategic games seems implausible under ambiguity [8-10].

To model situations with indeterminate payoffs, one kind of games, known as Bayesian games [11], is constructed based on the assumption that a player's belief regarding either the other players' types or their strategy choices is accurate. This means that the player is assumed to have a precise probability distribution over the consequences of a strategy profile. Another kind of games, called fuzzy games $[8,12$, 13], assumes instead that the payoffs are fuzzy. This kind of model usually interprets ambiguity as cases where decision makers are uncertain about the probability of each possibility. It is worth noting that players' optimistic attitudes are not considered in such fuzzy games.

Unlike these two approaches, some researchers have tried to apply some original ideas taken from decision theory to games [14-18]. To be more specific, they use nonadditive beliefs (capacities) [19] or multiple prior model (a set of probabilities) to represent ambiguity. Nevertheless, these kinds of work still assume that the consequences in a game are accurate. They thus cannot adequately handle the problem concerning ambiguous consequences and players' optimistic attitudes. More recently, researchers [20] have proposed a theoretic framework to study games with ambiguous payoffs, where only the case of ambiguity aversion in an interactive situation has been considered.

The aim of this paper is to propose a game-theoretic framework that can deal with imprecise probability theories. Using this theory, we first construct a decision model, in which decision makers are assumed to employ the Hurwicz criterion [21] (a well-known rule that has been used to 
model decisions under uncertainty $[22,23])$ to determine a preference ordering over actions given the interval-valued expected utilities and optimistic attitudes.

Based on this decision-theoretic framework, we then propose a game model, that is, the ambiguous game, which relaxes the assumption that a player's belief about the consequences of a strategy profile should be precise. In contrast with those frameworks mentioned above, our model allows a player's belief about the consequence or payoff of each strategy profile to be represented by imprecise probabilities. More importantly, our approach can also be used to model situations where the consequence or payoff of each strategy profile may possibly be ambiguous, and players may have different optimistic attitudes towards ambiguity.

The remainder of this paper is organized as follows. Section 2 briefly reviews some basic concepts and notations of Dempster-Shafer theory, expected utility intervals defined by D-S theory, and the Hurwicz criterion. Section 3 describes the basic elements of strategic games with ambiguous payoffs and investigates the conditions under which the degrees in beliefs regarding possible consequences and optimistic attitudes influence the solutions of an ambiguous game. Section 4 illustrates our approach by considering a scenario concerning allocating resource of defending and attacking in military contexts. Section 5 discusses some related research on the issue of ambiguous games. Finally, in Section 6, we conclude the paper and provide some thoughts about future work.

\section{Preliminaries}

This section recaps Dempster-Shafer theory [24], the expected utility interval based on this theory [25], and the Hurwicz criterion [21].

Definition 1. Let $\Theta$ be a frame of discernment (i.e., the set of states of the world).

(i) A function $m: 2^{\Theta} \rightarrow[0,1]$ is called a basic probability assignment or a mass function over $\Theta$ if $m(\emptyset)=0$ and $\sum_{A \subseteq \Theta} m(A)=1$. If $m(A)>0$, then $A$ is said to be a focal element. A mass function $m$ is called a simple mass function over $\Theta$ if $m\left(A_{0}\right)=s$, $m(\Theta)=1-s$, where $A_{0} \subset \Theta$ and $0 \leq s \leq 1$. In this case, we call $s$ a focal mass value.

(ii) A function Bel : $2^{\Theta} \rightarrow[0,1]$, defined as follows, is a belief function over $\Theta$ :

$$
\operatorname{Bel}(A)=\sum_{B \subseteq A} m(B) .
$$

By formula (1), the belief function (called a simple support function) induced by a simple mass function over $\Theta$ is given as follows:

$$
\operatorname{Bel}(A)= \begin{cases}0 & \text { if } A_{0} \not \subset A, \\ s & \text { if } A_{0} \subset A \neq \Theta . \\ 1 & \text { if } A=\Theta .\end{cases}
$$

The mass function is one of the most important concepts in Dempster-Shafer theory. There exists a natural interpretation of this concept proposed by Shafer and Tversky [26], which interprets a mass function as the degree of reliability for encoding a piece of evidence. To illustrate this, suppose that we randomly draw a ball from an urn containing 300 balls, and we know that 100 of them are red, and the remaining balls might be red $(r)$, blue $(b)$, or green $(g)$. How should we model such a situation where we only have partial information about the proportion of the balls? According to the reliability interpretation, it can be modeled by a simple mass function: $m(\{r\})=1 / 3$, and $m(\Theta)=2 / 3$, where $\Theta=$ $\{r, b, g\}$. The mass value $1 / 3$ encodes that there are 100 balls which are red in the urn, and the value $2 / 3$ encodes that the remaining 200 balls might be red, blue, or green. It should be noted that $m(\{b\})=m(\{g\})=0$, since there is no information concerning the amount of blue and green balls in the urn.

Note that a probability function can be regarded as a special case of mass function. In fact, if $m(A)>0$ and $\sum m(A)=1$, where $A$ is a singleton (i.e., $|A|=1$ ), then $m$ is indeed a probability function because $m(A)=\operatorname{Bel}(A)=$ $p(A)$. Nevertheless, we have in general that $\operatorname{Bel}(A) \leq p(A)$. That is, the belief function can be interpreted as the lower bound of probability function (i.e., the worst case estimate for probability). Given a simple mass function with support degree $s$ to the focal element $A$, then, $m(A)=\operatorname{Bel}(A)=s$. Thus, we can interpret the focal mass value $s$ as the lower probability of focal element $A$.

The foregoing example shows that Dempster-Shafer theory can represent ambiguous evidence or information in a sensible way. In particular, the simple mass functions (basic mass functions) can be used to model some kinds of ambiguity including the cases of total ignorance and partial information. Regarding the former case, we can assume the support degree $s$ to be zero; that is, $m(A)=0, A \neq \Theta$, and $m(\Theta)=1$, which suitably describes the situation of total ignorance. In this case, we call $m$ a vacuous mass function. To represent the case of partial information, let support degree $s$ be 1 to a specific subset of the frame of discernment; that is, $m(A)=1, A \neq \Theta$. Such a mass function (called $a$ pseudovacuous mass function) can properly model the case of partial information, where we can only be certain that the true event belongs to a subset of the frame of discernment based on the available information.

Now, consider the decision situations, where a decision maker cannot identify the exact consequence that an action will result in, although he can specify the utilities of those possible consequences. In addition, it may be the case that he cannot even determine the probabilities associated with those possible consequences. Using the terminology of DempsterShafer theory, the decision maker can only represent such information in terms of a mass function defined over the set of possible consequences. Formally, we can define such decision situations as follows.

Definition 2. A decision problem under ambiguity (or called an ambiguity decision problem) is a 4-tuple of $(A, \Theta, M, u)$, where

(i) $A=\left\{a_{1}, \ldots, a_{n}\right\}$ is the set of all actions;

(ii) $\Theta=\left\{c_{1}, \ldots, c_{k}\right\}$ is the set of all of consequences of actions; 
(iii) $M=\left\{m_{1}, \ldots, m_{n}\right\}$, where $m_{i}(i=1, \ldots, n)$ is the mass function over a set $\Theta_{i}(\subset \Theta)$, the set of the consequences that action $a_{i} \in A$ could lead to;

(iv) $u$ is a utility function; that is, $u: \Theta \rightarrow \mathbb{R}$, where $\mathbb{R}$ is the set of real numbers.

Then, based on the concept of mass functions, the pointvalued expected utility formula can be extended to the context of ambiguity by defining the notion called expected utility interval [25].

Definition 3. Given an ambiguity decision problem $(A, \Theta, M, u)$, for action $a_{i} \in A$, its expected utility interval is given by $\operatorname{EUI}\left(a_{i}\right)=\left[\underline{U}\left(a_{i}\right), \bar{U}\left(a_{i}\right)\right]$, where

$$
\begin{aligned}
& \underline{U}\left(a_{i}\right)=\sum_{B \subseteq \Theta_{i}} m_{i}(B) \min \left\{u\left(c_{i}\right) \mid c_{i} \in B\right\}, \\
& \bar{U}\left(a_{i}\right)=\sum_{B \subseteq \Theta_{i}} m_{i}(B) \max \left\{u\left(c_{i}\right) \mid c_{i} \in B\right\} .
\end{aligned}
$$

We cannot obtain a complete preference ordering from an expected utility interval. To address the problem, we can employ a well-known decision rule called the Hurwicz criterion [21], which is given as follows:

$$
\Xi\left(a_{i}\right)=\left(1-\omega_{i}\right) \underline{U}\left(a_{i}\right)+\omega_{i} \bar{U}\left(a_{i}\right),
$$

where the pointed value $\Xi\left(a_{i}\right)$ is called the generalized expected utility or payoff of action $a_{i}$ and the index $\omega_{i} \in[0,1]$ can be interpreted as a degree of optimism, which reflects a decision attitude. Particularly, If $1 / 2<\omega_{i} \leq 1$, then the decision attitude is optimistic. In contrast, the decision attitude is pessimistic if $0 \leq \omega_{i}<1 / 2$. And the decision attitude is neutral if $\omega_{i}=1 / 2$.

More specifically, the Hurwicz criterion says that, for each action, a decision maker takes a weighted average of the purely optimistic and purely pessimistic value by the degree of optimism. In particular, if $\omega_{i}=0$, then $\Xi\left(a_{i}\right)=\underline{U}\left(a_{i}\right)$. This means that the decision maker has a purely pessimistic attitude and chooses the alternative that has the best worst. This strategy is often called the maximin strategy. In contrast, if $\omega_{i}=1$, then $\Xi\left(a_{i}\right)=\bar{U}\left(a_{i}\right)$. This means that the decision maker has a purely optimistic attitude and selects the action that has the best best. This strategy is often called the maximax strategy.

Using this criterion, the preference can then be determined as

$$
a_{i} \geq a_{j} \Longleftrightarrow \Xi\left(a_{i}\right) \geq \Xi\left(a_{j}\right) .
$$

It is easy to see that the preference ordering defined as (5) is complete.

\section{Game Definition}

This section presents a new game-theoretic framework for games with ambiguous consequences and then introduces a solution concept for solving such games.
TABle 1: Payoff matrix of a $2 \times 2$ game.

\begin{tabular}{lll}
\hline & $b_{1}$ & $b_{2}$ \\
\hline$a_{1}$ & $\Theta_{1}^{\left(a_{1}, b_{1}\right)}, \Theta_{2}^{\left(a_{1}, b_{1}\right)}$ & $\Theta_{1}^{\left(a_{1}, b_{2}\right)}, \Theta_{2}^{\left(a_{1}, b_{2}\right)}$ \\
$a_{2}$ & $\Theta_{1}^{\left(a_{2}, b_{1}\right)}, \Theta_{2}^{\left(a_{2}, b_{1}\right)}$ & $\Theta_{1}^{\left(a_{2}, b_{2}\right)}, \Theta_{2}^{\left(a_{2}, b_{2}\right)}$ \\
\hline
\end{tabular}

Intuitively, games with ambiguous consequences are designed to depict cases where players only know that the possible consequences of each strategy profile lie in a certain set. For example, Table 1 is the payoff matrix of a $2 \times 2$ game, where $\Theta_{i}^{\left(a_{j}, b_{k}\right)}$ is the set of possible consequences associated with the strategy profile $\left(a_{j}, b_{k}\right)$ for player $i$. However, players are not sure which one of the consequence will occur and also what the probability of each consequence is. More precisely, for each strategy profile, the players can only use a mass function, rather than a probability measure, defined over the set of possible consequences to represent ambiguity concerning consequences. In particular, if mass functions are simple (see Definition 1), then the players are only required to determine the lower probability of some possible consequences within that set. In order to obtain the generalized expected utilities and a complete preference ordering, players need to assign a degree of optimism for each strategy profile. We can then formally define an ambiguous game as follows.

Definition 4. An ambiguous game denoted as $G$ is a tuple $G=$ $(N, A, \Theta, M, \Omega, U)$, where

(i) $N$ is a set of natural numbers, which denotes the set of players of the game;

(ii) $A=\left\{A_{i}\right\}_{i \in N}$, where $A_{i}$ denotes a finite set of pure strategies (or actions) for player $i$;

(iii) $\Theta=\left\{\Theta_{i}\right\}_{i \in N}$, where $\Theta_{i}=\left\{\Theta_{i}^{\rho} \mid \Theta_{i}^{\rho}\right.$ is the set of possible consequences associated with the pure strategy profile $\rho$ for player $i$;

(iv) $M=\left\{M_{i}\right\}_{i \in N}$, where $M_{i}=\left\{m_{i}^{\rho} \mid m_{i}^{\rho}\right.$ is the simple mass function over $\Theta_{i}^{\rho}$ associated with the pure strategy profile $\rho$ for player $i\}$;

(v) $\Omega=\left\{\Omega_{i}\right\}_{i \in N}$, where $\Omega_{i}=\left\{\omega_{i}^{\rho} \mid \omega_{i}^{\rho}\right.$ is the degree of optimism associated with the pure strategy profile $\rho$ for player $i\}$;

(vi) $U=\left\{u_{i}\right\}_{i \in N}$, where $u_{i}$ denotes the payoff function of player $i$ from the set of the all consequences to the set of real numbers; that is, $u_{i}: \bigcup_{i} \Theta_{i} \rightarrow \mathbb{R}$.

Given an ambiguous game, each player needs to compute the expected payoff interval associated with a pure strategy profile according to formulas (3). By applying the Hurwicz criterion, we can obtain the generalized expected payoffs of profiles. In particular, we have the following result.

Lemma 5. Given an ambiguous game $G=(N, A, \Theta, M, \Omega$, $U)$, suppose that, for any pure strategy profile $\rho, m_{i}^{\rho}\left(B_{i}^{\rho}\right)=$ $\xi_{i}^{\rho}, m_{i}^{\rho}\left(\Theta_{i}^{\rho}\right)=1-\xi_{i}^{\rho}, B_{i}^{\rho} \subset \Theta_{i}^{\rho}$, and $\omega_{i}^{\rho} \in \Omega_{i}$. Let $t_{i}^{\rho}=\min \left\{u_{i}\left(c_{i}\right) \mid c_{i} \in B_{i}^{\rho}\right\}, T_{i}^{\rho}=\max \left\{u_{i}\left(c_{i}\right) \mid c_{i} \in B_{i}^{\rho}\right\}, y_{i}^{\rho}=$ $\min \left\{u_{i}\left(c_{i}\right) \mid c_{i} \in \Theta_{i}^{\rho}\right\}$, and $Y_{i}^{\rho}=\max \left\{u_{i}\left(c_{i}\right) \mid c_{i} \in \Theta_{i}^{\rho}\right\}$, and let 
$\Xi_{i}^{\rho}$ be the generalized expected utility of the pure strategy profile $\rho$. Then,

$$
\begin{aligned}
\Xi_{i}^{\rho}= & \left(1-\omega_{i}^{\rho}\right)\left(\xi_{i}^{\rho} t_{i}^{\rho}+\left(1-\xi_{i}^{\rho}\right) y_{i}^{\rho}\right) \\
& +\omega_{i}^{\rho}\left(\xi_{i}^{\rho} T_{i}^{\rho}+\left(1-\xi_{i}^{\rho}\right) Y_{i}^{\rho}\right) .
\end{aligned}
$$

Proof. Since player $i$ has simple support function $m_{i}^{\rho}$, by formulas (3), the lower and upper expected payoffs of profile $\rho$ given mass function $m_{i}^{\rho}$ are given as follows:

$$
\begin{aligned}
& \underline{U}_{i}^{\rho}=\xi_{i}^{\rho} t_{i}^{\rho}+\left(1-\xi_{i}^{\rho}\right) y_{i}^{\rho}, \\
& \bar{U}_{i}^{\rho}=\xi_{i}^{\rho} T_{i}^{\rho}+\left(1-\xi_{i}^{\rho}\right) Y_{i}^{\rho} .
\end{aligned}
$$

By formula (4), we obtain the generalized expected utility of profile $\rho$ for player $i$ as formula (6), which establishes the required result.

The above lemma provides a way to calculate $\Xi_{i}^{\rho}$ associated with any pure strategy profile $\rho$ in an ambiguous game where mass functions are simple. In particular, if $m_{i}^{\rho}$ is a vacuous mass function, that is, $\xi_{i}^{\rho}=0$, then, by formula (6), we obtain that

$$
\Xi_{i}^{\rho}=\left(1-\omega_{i}^{\rho}\right) y_{i}^{\rho}+\omega_{i}^{\rho} Y_{i}^{\rho} .
$$

In addition, if $m_{i}^{\rho}$ is a pseudovacuous mass function, that is, $\xi_{i}^{\rho}=1$, then we have

$$
\Xi_{i}^{\rho}=\left(1-\omega_{i}^{\rho}\right) t_{i}^{\rho}+\omega_{i}^{\rho} T_{i}^{\rho} .
$$

It thus follows that we can transform an ambiguous game into a strategic game defined by classic game theory $[27,28]$. As such, we can derive a normal form game with precise payoffs from an ambiguous game. More precisely, given an ambiguous game $G$ we can turn it into a strategic game $G^{\prime}=$ $\left(N, A, U^{\prime}\right)$, where $U^{\prime}=\left\{u_{i}^{\prime}\right\}_{i \in N}$ and $u_{i}^{\prime}(\rho)=\Xi_{i}^{\rho}$.

Definition 6. Given an ambiguous game $G=(N, A, \Theta, M$, $\Omega, U)$, a strategic game, denoted by $G^{\prime}=\left(N, A, U^{\prime}\right)$ (since $u_{i}^{\prime}(\rho)=\Xi_{i}^{\rho}$ by formula (6), the payoff $u^{\prime}$ depends on $\omega_{i}^{\rho}$ and $\left.\xi_{i}^{\rho}\right)$, is called an induced game of $G$, if the element of its payoff matrix is obtained by using formula (6) based on $G$.

As usual, a mixed strategy $\alpha_{i}$ for player $i$ is defined as a probability distribution over the pure strategy set $A_{i}$. We denote $\alpha_{i}\left(a_{i}\right)$ as the probability assigned by the mixed strategy $\alpha_{i}$ to player $i$ 's pure strategy $a_{i} \in A_{i}$. Let $\Xi_{i}(\alpha)$ be player $i$ 's generalized expected payoff given by the mixed-strategy profile $\alpha=\left(\alpha_{i}, \alpha_{-i}\right)$. A pure strategy can then be regarded as a degenerate case of a mixed strategy, in which that pure strategy is assigned with probability 1 .

Given an ambiguous game $G=(N, A, \Theta, M, \Omega, U)$ and a mixed-strategy profile $\alpha$, we can compute player $i$ 's generalized expected payoff to $\alpha$ as follows:

$$
\Xi_{i}(\alpha)=\sum_{\rho}\left(\prod_{i} \alpha_{i}\left(a_{i}\right)\right) \Xi_{i}^{\rho},
$$

where $a_{i} \in A_{i}$ is the element of $\rho$ and $\Xi_{i}^{\rho}$ is given by (6).
Definition 7. A mixed-strategy profile $\alpha^{*}=\left(\alpha_{i}^{*}, \alpha_{-i}^{*}\right)$ in the ambiguous game $G=(N, A, \Theta, M, \Omega, U)$ is an ambiguous mixed-strategy equilibrium, if for all $i \in N$, for every mixedstrategy $\alpha_{i}$ of player $i, \Xi_{i}\left(\alpha_{i}^{*}, \alpha_{-i}^{*}\right) \geq \Xi_{i}\left(\alpha_{i}, \alpha_{-i}^{*}\right)$.

Theorem 8. Given ambiguous game $G=(N, A, \Theta, M, \Omega, U)$, suppose that $\alpha^{*}$ is an ambiguous mixed-strategy equilibrium of game $G$. Then $\alpha^{*}$ is an ambiguous equilibrium of $G$ if and only if $\alpha^{*}$ is a mixed-strategy Nash equilibrium of its induced game $G^{\prime}=\left(N, A, U^{\prime}\right)$.

Proof. By Definition 6 and formula (10), for any mixedstrategy profile $\alpha=\left(\alpha_{i}, \alpha_{-i}\right)$, we have that

$$
\Xi_{i}\left(\alpha_{i}, \alpha_{-i}\right)=E_{i}\left(\alpha_{i}, \alpha_{-i}\right)
$$

where $E_{i}\left(\alpha_{i}, \alpha_{-i}\right)$ is player $i$ 's expected payoff given by mixedstrategy profile $\alpha$ in game $G^{\prime}$. Let $\alpha^{*}$ be a mixed-strategy Nash equilibrium of game $G^{\prime}$. Then, for any $\alpha_{i} \neq \alpha_{i}^{*}$, we have

$$
E_{i}\left(\alpha_{i}^{*}, \alpha_{-i}^{*}\right) \geq E_{i}\left(\alpha_{i}, \alpha_{-i}^{*}\right) .
$$

This implies that, for any $\alpha_{i} \neq \alpha_{i}^{*}$, we have

$$
\Xi_{i}\left(\alpha_{i}^{*}, \alpha_{-i}^{*}\right) \geq \Xi_{i}\left(\alpha_{i}, \alpha_{-i}^{*}\right) .
$$

Then, according to Definition 7, the mixed-strategy profile $\alpha^{*}$ is an ambiguous equilibrium of $G$.

If $\alpha^{*}$ is an ambiguous equilibrium of $G$, then we can show that $\alpha^{*}$ is also a mixed-strategy Nash equilibrium of $G^{\prime}$ in a similar way.

Recall from the well-known result proved by Nash that there always exists at least one mixed-strategy Nash equilibrium for any finite game [28]. It follows immediately from Theorem 8 that any ambiguous game has at least one ambiguous equilibrium, since its induced game has at least one Nash equilibrium.

Essentially, Theorem 8 provides an approach to find the ambiguous mixed-strategy equilibria of an ambiguous game. Given an ambiguous game, we can obtain the induced game with focal mass values using formulas (6) and (10) and then find the mixed-strategy Nash equilibria of the induced game, which are also the ambiguous mixed-strategy equilibria of the original ambiguous game. In this sense, we can find ambiguous mixed strategy equilibria when focal mass values $\xi_{i}^{\rho}$ are given. Therefore, the set of ambiguous mixed-strategy equilibria can be regarded as a value of a function, where the variables are the focal mass values.

It is worth noting that in our model players' payoffs are allowed to be ambiguous, while the traditional games always assume precise payoffs. Within our framework, the point-valued payoffs in the induced game are computed from an interval-valued payoff of the original ambiguous game. Nevertheless, our approach can capture the idea of ambiguity aversion under interactive situations, which is a missing feature of the traditional game-theoretic approaches.

Next, we study how the focused masses may influence the outcomes (solutions) of an ambiguous game. Suppose that every focal mass value $\xi_{i}^{\rho}$ in Theorem 8 is an unknown 
variable. The following theorem demonstrates how to find the maximum value of function $\Xi_{i}\left(\xi_{i}^{\rho}, \ldots, \xi_{i}^{\rho^{\prime}}\right)$ given a mixedstrategy profile.

Theorem 9. Given an ambiguous game $(N, A, \Theta, M, \Omega, U)$, suppose that $m_{i}^{\rho}\left(B_{i}^{\rho}\right)=\xi_{i}^{\rho}, m_{i}^{\rho}\left(\Theta_{i}^{\rho}\right)=1-\xi_{i}^{\rho}, B_{i}^{\rho} \subset \Theta_{i}^{\rho}$, $i \in N$, and $\omega_{i}^{\rho}, \ldots, \xi_{i}^{\rho^{\prime}} \in \Omega_{i}$. Let $t_{i}^{\rho}=\min \left\{u_{i}\left(c_{i}\right) \mid c_{i} \in B_{i}^{\rho}\right\}$, $T_{i}^{\rho}=\max \left\{u_{i}\left(c_{i}\right) \mid c_{i} \in B_{i}^{\rho}\right\}, y_{i}^{\rho}=\min \left\{u_{i}\left(c_{i}\right) \mid c_{i} \in \Theta_{i}^{\rho}\right\}$, and $Y_{i}^{\rho}=\max \left\{u_{i}\left(c_{i}\right) \mid c_{i} \in \Theta_{i}^{\rho}\right\}$. Given a mixed-strategy profile $\alpha$, let $\Xi_{i}$ be player i's generalized expected utility to $\alpha$. Moreover, define $\beta_{i}^{\rho}$ as follows:

$$
\beta_{i}^{\rho}=\frac{t_{i}^{\rho}-y_{i}^{\rho}}{\left(Y_{i}^{\rho}-T_{i}^{\rho}\right)+\left(t_{i}^{\rho}-y_{i}^{\rho}\right)} .
$$

Then, the following assertions hold:

(i) if $\omega_{i}^{\rho}<\beta_{i}^{\rho}$, then function $\Xi_{i}\left(\xi_{i}^{\rho}, \ldots, \xi_{i}^{\rho^{\prime}}\right)$ is strictly increasing in the coordinate $\xi_{i}^{\rho}$;

(ii) if $\omega_{i}^{\rho}>\beta_{i}^{\rho}$, then the function $\Xi_{i}\left(\xi_{i}^{\rho}, \ldots, \xi_{i}^{\rho^{\prime}}\right)$ is strictly decreasing in the coordinate $\xi_{i}^{\rho}$;

(iii) if $\omega_{i}^{\rho}=\beta_{i}^{\rho}$, then the function $\Xi_{i}\left(\xi_{i}^{\rho}, \ldots, \xi_{i}^{\rho^{\prime}}\right)$ is independent of the coordinate $\xi_{i}^{\rho}$;

(iv) the function $\Xi_{i}\left(\xi_{i}^{\rho}, \ldots, \xi_{i}^{\rho^{\prime}}\right)$ has its maximum at $\left(\widehat{\xi}_{i}^{\rho}, \ldots, \widehat{\xi}_{i}^{\rho^{\prime}}\right)$, where

$$
\hat{\xi}_{i}^{\rho}= \begin{cases}1 & \text { if } \omega_{i}^{\rho}<\beta_{i}^{\rho}, \\ 0 & \text { if } \omega_{i}^{\rho}>\beta_{i}^{\rho}, \\ x, x \in[0,1] & \text { if } \omega_{i}^{\rho}=\beta_{i}^{\rho} .\end{cases}
$$

Proof. Firstly, we show that $0 \leq \beta_{i}^{\rho} \leq 1$. Since $B_{i}^{\rho} \subset \Theta_{i}^{\rho}$, we have $y_{i}^{\rho} \leq t_{i}^{\rho} \leq T_{i}^{\rho}<Y_{i}^{\rho}$ or $y_{i}^{\rho}<t_{i}^{\rho} \leq T_{i}^{\rho} \leq Y_{i}^{\rho}$, which implies that $0 \leq \beta_{i}^{\rho} \leq 1$.

For any mixed-strategy profile $\alpha$, by formulas (6) and (10), we have

$$
\begin{aligned}
& \Xi_{i}\left(\xi_{i}^{\rho}, \ldots, \xi_{i}^{\rho^{\prime}}\right) \\
& =\sum_{\rho}\left(\prod_{i} \alpha_{i}\left(a_{i}\right)\right) \Xi_{i}^{\rho}, \\
& =\sum_{\rho}\left(\prod_{i} \alpha_{i}\left(a_{i}\right)\right)\left(\left(1-\omega_{i}^{\rho}\right)\left(\xi_{i}^{\rho} t_{i}^{\rho}+\left(1-\xi_{i}^{\rho}\right) y_{i}^{\rho}\right)\right. \\
& \left.+\omega_{i}^{\rho}\left(\xi_{i}^{\rho} T_{i}^{\rho}+\left(1-\xi_{i}^{\rho}\right) Y_{i}^{\rho}\right)\right) \\
& =\sum_{\rho}\left(\prod_{i} \alpha_{i}\left(a_{i}\right)\right)\left(\xi _ { i } ^ { \rho } \left(\omega_{i}^{\rho}\left(T_{i}^{\rho}-t_{i}^{\rho}-Y_{i}^{\rho}+y_{i}^{\rho}\right)\right.\right. \\
& \left.+t_{i}^{\rho}-y_{i}^{\rho}\right)+\omega_{i}^{\rho} Y_{i}^{\rho} \\
& \left.+\left(1-\omega_{i}^{\rho}\right) y_{i}^{\rho}\right) .
\end{aligned}
$$

Let

$$
\begin{aligned}
f_{i}^{\rho}\left(\xi_{i}^{\rho}\right)= & \xi_{i}^{\rho}\left(\omega_{i}^{\rho}\left(T_{i}^{\rho}-t_{i}^{\rho}-Y_{i}^{\rho}+y_{i}^{\rho}\right)+t_{i}^{\rho}-y_{i}^{\rho}\right) \\
& +\omega_{i}^{\rho} Y_{i}^{\rho}+\left(1-\omega_{i}^{\rho}\right) y_{i}^{\rho} .
\end{aligned}
$$

Clearly, $f_{i}^{\rho}\left(\xi_{i}^{\rho}\right)$ is linear.

If $\omega_{i}^{\rho}<\beta_{i}^{\rho}$, then

$$
\omega_{i}^{\rho}\left(T_{i}^{\rho}-t_{i}^{\rho}-Y_{i}^{\rho}+y_{i}^{\rho}\right)+t_{i}^{\rho}-y_{i}^{\rho}>0 .
$$

In this case, $f_{i}^{\rho}\left(\xi_{i}^{\rho}\right)$ is strictly increasing, and thus $\Xi_{i}\left(\xi_{i}^{\rho}\right.$, $\left.\ldots, \xi_{i}^{\rho^{\prime}}\right)$ is also strictly increasing in the coordinate $\xi_{i}^{\rho}$.

If $\omega_{i}^{\rho}>\beta_{i}^{\rho}$, then

$$
\omega_{i}^{\rho}\left(T_{i}^{\rho}-t_{i}^{\rho}-Y_{i}^{\rho}+y_{i}^{\rho}\right)+t_{i}^{\rho}-y_{i}^{\rho}<0 .
$$

In this case, $f_{i}^{\rho}\left(\xi_{i}^{\rho}\right)$ is strictly decreasing, and accordingly $\Xi_{i}\left(\xi_{i}^{\rho}, \ldots, \xi_{i}^{\rho^{\prime}}\right)$ is strictly decreasing in the coordinate $\xi_{i}^{\rho}$ as well.

Finally, if $\omega_{i}^{\rho}=\beta_{i}^{\rho}$, then

$$
\omega_{i}^{\rho}\left(T_{i}^{\rho}-t_{i}^{\rho}-Y_{i}^{\rho}+y_{i}^{\rho}\right)+t_{i}^{\rho}-y_{i}^{\rho}=0,
$$

which means that $\Xi_{i}\left(\xi_{i}^{\rho}, \ldots, \xi_{i}^{\rho^{\prime}}\right)$ is independent of the coordinate $\xi_{i}^{\rho}$. In this case, $f_{i}^{\rho}\left(\xi_{i}^{\rho}\right)=\beta_{i}^{\rho} Y_{i}^{\rho}+\left(1-\beta_{i}^{\rho}\right) y_{i}^{\rho}$, and thus we have

$$
\Xi_{i}\left(\xi_{i}^{\rho}, \ldots, \xi_{i}^{\rho^{\prime}}\right)=\sum_{\rho}\left(\prod_{i} \alpha_{i}\left(a_{i}\right)\right)\left(\beta_{i}^{\rho} Y_{i}^{\rho}+\left(1-\beta_{i}^{\rho}\right) y_{i}^{\rho}\right) .
$$

Since function $f_{i}^{\rho}\left(\xi_{i}^{\rho}\right)$ is linear, $\Xi_{i}\left(\xi_{i}^{\rho}, \ldots, \xi_{i}^{\rho^{\prime}}\right)$ has its maximum if each $f\left(\xi_{i}^{\rho}\right)$ achieves its maximum. Thus, the function has its maximum at $\left(\hat{\xi}_{i}^{\rho}, \ldots, \hat{\xi}_{i}^{\rho^{\prime}}\right)$, where $\hat{\xi}_{i}^{\rho}=1$, if $\omega_{i}^{\rho}<\beta_{i}^{\rho}, \hat{\xi}_{i}^{\rho}=0$ if $\omega_{i}^{\rho}>\beta_{i}^{\rho}$, and $\widehat{\xi}_{i}^{\rho}=x$, where $x \in[0,1]$, if $\omega_{i}^{\rho}=\beta_{i}^{\rho}$.

As shown in Theorem 9, we have established the relationship between focal mass values, optimistic attitudes, and the generalized expected payoffs given by any mixed-strategy profile. As a result, this theorem demonstrates what a player should do under an ambiguous interactive situation, where he/she only needs to decide his/her $\beta$-values and degree of optimism. More specifically, item (i) shows that, for any player $i$, if $\beta_{i}^{\rho}$ is more than his/her corresponding degrees of optimism $\omega_{i}^{\rho}$, then the bigger the focal mass value (i.e., the less ambiguity), the bigger the generalized expected payoff and thus the better for player $i$. In contrast, item (ii) indicates that, if $\beta_{i}^{\rho}$ is less than his/her corresponding degrees of optimism $\omega_{i}^{\rho}$, then the more ambiguity the better for player $i$. And item (iii) says that if $\beta_{i}^{\rho}$ is equal to his/her corresponding degrees of optimism $\omega_{i}^{\rho}$, then player $i$ could assign any focal mass value for the focal element of mass function $m_{i}^{\rho}$. In addition, item (iv) identifies the conditions under which function $\Xi_{i}$ has a maximum value. 
It is important to note that Theorem 9 holds for any mixed-strategy profile. In light of these results, it seems reasonable to say that there is a close relationship between the focal mass values and the equilibria of an ambiguous game (to find the equilibria of an ambiguous game, players' focal mass values and degrees of optimism are required to be common knowledge among players; however, Theorem 9 holds even if a player only knows his/her own mass values and degrees of optimism).

The generalized expected utility $\Xi_{i}$ is calculated according to the Hurwicz criterion, which depends on the attitude of player $i$ in an ambiguous game.

Corollary 10. Under the conditions given in Theorem 9, the following assertions hold:

(i) if $\omega_{i}^{\rho}<\beta_{i}^{\rho}$, and player $i$ switches from his/her $\omega_{i}^{\rho}$ to $\omega_{i}^{\prime \rho}$, $\omega_{i}^{\prime \rho}>\beta_{i}^{\rho}$, then $\Xi_{i}\left(\xi_{i}^{\rho}, \ldots, \xi_{i}^{\rho^{\prime}}\right)>\Xi_{i}\left(\xi_{i}^{\prime \rho}, \ldots, \xi_{i}^{\rho^{\prime}}\right)$, where $\xi_{i}^{\prime \rho}<\xi_{i}^{\rho}$

(ii) if $\omega_{i}^{\rho}>\beta_{i}^{\rho}$, and player $i$ switches from his/her $\omega_{i}^{\rho}$ to $\omega_{i}^{\prime \rho}$, $\omega_{i}^{\prime \rho}<\beta_{i}^{\rho}$, then $\Xi_{i}\left(\xi_{i}^{\rho}, \ldots, \xi_{i}^{\rho^{\prime}}\right)>\Xi_{i}\left(\xi_{i}^{\prime \rho}, \ldots, \xi_{i}^{\rho^{\prime}}\right)$, where $\xi_{i}^{\prime \rho}>\xi_{i}^{\rho}$.

Proof. Since $\omega_{i}^{\prime \rho}<\beta_{i}^{\rho}$, by item (ii) of Theorem 9, function $\Xi_{i}\left(\xi_{i}^{\rho}, \ldots, \xi_{i}^{\rho^{\prime}}\right)$ is strictly decreasing in the coordinate $\xi_{i}^{\rho}$. This means that the smaller the focal mass value, the bigger the generalized expected payoff to any mixed-strategy profile. Thus, as a rational player, player $i$ will try to get less information so that he/she will obtain a bigger generalized expected payoff. That is, we have $\xi_{i}^{\prime \rho}<\xi_{i}^{\rho}$. On the other hand, since $\omega_{i}^{\rho}<\beta_{i}^{\rho}$, by item (i) of Theorem 9, function $\Xi_{i}\left(\xi_{i}^{\rho}, \ldots, \xi_{i}^{\rho^{\prime}}\right)$ is strictly increasing in the coordinate $\xi_{i}^{\rho}$. So, we have $\Xi_{i}\left(\xi_{i}^{\rho}, \ldots, \xi_{i}^{\rho^{\prime}}\right)>\Xi_{i}\left(\xi_{i}^{\prime \rho}, \ldots, \xi_{i}^{\rho^{\prime}}\right)$. This completes the proof of item (i). Similarly, we can establish item (ii).

Corollary 10 investigates what happens when a player is too optimistic or pessimistic about a strategy profile. If $\omega_{i}^{\rho}<$ $\beta_{i}^{\rho}$ and player $i$ switches from his/her $\omega_{i}^{\rho}$ to $\omega_{i}^{\prime \rho}$ with $\omega_{i}^{\prime \rho}>\beta_{i}^{\rho}$, then player $i$ is too optimistic. In contrast, if if $\omega_{i}^{\rho}>\beta_{i}^{\rho}$ and player $i$ switches from his/her $\omega_{i}^{\rho}$ to $\omega_{i}^{\prime \rho}$ with $\omega_{i}^{\prime \rho}<\beta_{i}^{\rho}$, then we say that player $i$ is too pessimistic. The corollary shows that any player's generalized expected payoff will be decreasing if he/she is too optimistic or pessimistic.

\section{Example: A Defending and Attacking Game}

In this section, we illustrate our game-theoretic framework using a strategic situation between a defender and an attacker (both could be a team).

4.1. Problem Description. In this problem, the attacker needs to allocate his/her available resources, for example, personnel and weapons, to attack some targets chosen from three international airports in a country, while the defender needs to allocate his available resources to prevent attacks. Suppose that the possible attack effects or consequences can be divided into 11 different scales from the 1st one being the best attack effect (denoted by $c_{1}$ ) to the 11th one being the worst attack effect (denoted by $c_{11}$ ). Clearly, the attacker's preference ranking over the set of the possible attack effects or consequences is from $c_{1}$ to $c_{11}$, while the defender's preference ranking over that set is reversed.

Moreover, suppose that there are two kinds of information available in this case. First, public information is known by both the defender and the attacker, such as the average passenger flows, the architectures, the flight schedules of these three airports, the regular safety inspections, and ordinary defending and attacking methods. By contrast, private information of one player might not be available to his opponent. For example, special defending and attacking resources can be regarded as private information, such as irregular patrols, special attacking methods and skills, and the allocation of attackers. In addition, there are some random factors in the game environment that might have impact on the attack effects, which are not controlled by neither the defender nor the attacker during the possible attacks, such as the cooperation abilities of related departments to deal with emergences, the defending skills of unarmed people, and the weather.

As such, it seems unrealistic for both the defender and the attacker to specify the exact consequence (or precise payoff) of a pure strategy profile. Nevertheless, based upon public information and relevant knowledge, they are certain that the possible consequences of each pure strategy profile $\rho$ lie in a set of $\Theta_{i}^{\rho}$, a subset of the set of all the possible attack effects. Due to limited information, the defender and the attacker can only identify the lower probabilities (the focal mass values), rather than exact probabilities, of some possible consequences of the pure strategy profile $\rho$. Furthermore, given such mass values, they both hold an optimistic attitude for each pure strategy profile that determines a generalized expected payoff under the profile.

4.2. Game Model. In this case, we write the pure strategies of the defender and the attacker as $a_{1}, a_{2}$, and $a_{3}$, which mean that the defender and the attacker use their full resources to prevent and attack the airports $a_{1}, a_{2}$, and $a_{3}$, respectively. According to public information, the ambiguity concerning consequences of each pure strategy profile $\rho$ is represented by a simple mass function $m_{1}^{\rho}$ for the defender and $m_{2}^{\rho}$ for the attacker.

By applying Definition 4, this interactive situation can then be modeled as an ambiguous game of $G=(N, A, \Theta, M, \Omega, U)$, where

(i) $N=\{1,2\}$ is the player set, where player 1 is the defender and player 2 is the attacker;

(ii) $A=\left\{A_{1}, A_{2}\right\}$ is the pure strategy set, where $A_{1}=$ $A_{2}=\left\{a_{1}, a_{2}, a_{3}\right\}$

(iii) $\Theta=\left\{\Theta_{1}^{\rho}, \Theta_{2}^{\rho}\right\}$ is the consequence set, where $\Theta_{1}^{\rho}$ and $\Theta_{2}^{\rho}$ are the sets of attack effects given pure strategy profile $\rho$, which are given as row 3 in Tables 2 and 3, respectively, and $\bigcup_{\rho} \Theta_{1}^{\rho}=\bigcup_{\rho} \Theta_{2}^{\rho}=\left\{c_{1}, c_{2}, \ldots, c_{11}\right\}$, where $c_{i}$ is an attack effect, $i=1,2, \ldots, 11$; 
TABLE 2: Important elements of the ambiguous game for the defender.

\begin{tabular}{lccccc}
\hline & $B_{1}^{\rho}$ & $\Theta_{1}^{\rho}$ & $\xi_{1}^{\rho}$ & $\beta_{1}^{\rho}$ & $\omega_{1}^{\rho}$ \\
\hline$\rho=\left(a_{1}, a_{1}\right)$ & $\left\{c_{10}, c_{11}\right\}$ & $\left\{c_{7}, \ldots, c_{11}\right\}$ & 0.7 & 1 & $1 / 3$ \\
$\rho=\left(a_{1}, a_{2}\right)$ & $\left\{c_{5}\right\}$ & $\left\{c_{4}, \ldots, c_{7}\right\}$ & 0.4 & $1 / 3$ & $1 / 2$ \\
$\rho=\left(a_{1}, a_{3}\right)$ & $\left\{c_{3}\right\}$ & $\left\{c_{1}, \ldots, c_{5}\right\}$ & 0.8 & $1 / 2$ & $1 / 2$ \\
$\rho=\left(a_{2}, a_{1}\right)$ & $\left\{c_{1}, c_{2}\right\}$ & $\left\{c_{1}, \ldots, c_{5}\right\}$ & 0.3 & 0 & $4 / 5$ \\
$\rho=\left(a_{2}, a_{2}\right)$ & $\left\{c_{8}\right\}$ & $\left\{c_{6}, \ldots, c_{9}\right\}$ & 0.5 & $2 / 3$ & $1 / 2$ \\
$\rho=\left(a_{2}, a_{3}\right)$ & $\left\{c_{3}\right\}$ & $\left\{c_{1}, \ldots, c_{4}\right\}$ & 0.6 & $2 / 3$ & $4 / 5$ \\
$\rho=\left(a_{3}, a_{1}\right)$ & $\left\{c_{1}\right\}$ & $\left\{c_{1}, \ldots, c_{5}\right\}$ & 0.6 & $1 / 2$ & $2 / 3$ \\
$\rho=\left(a_{3}, a_{2}\right)$ & $\left\{c_{5}\right\}$ & $\left\{c_{4}, c_{5}, c_{6}\right\}$ & 0.7 & $1 / 2$ & $1 / 2$ \\
$\rho=\left(a_{3}, a_{3}\right)$ & $\left\{c_{9}\right\}$ & $\left\{c_{7}, \ldots, c_{11}\right\}$ & 0.8 & $1 / 2$ & $1 / 2$ \\
\hline
\end{tabular}

(iv) $M=\left\{M_{1}, M_{2}\right\}$ is the mass function set, where $M_{1}=\left\{m_{1}^{\rho}\right\}, M_{2}=\left\{m_{2}^{\rho}\right\}, m_{1}^{\rho}$ and $m_{2}^{\rho}$ are simple mass functions over $\Theta_{1}^{\rho}$ and $\Theta_{2}^{\rho}$, and the focal elements are $B_{1}^{\rho}$ and $B_{2}^{\rho}$ with focal mass values $\xi_{1}^{\rho}$ and $\xi_{2}^{\rho}$, respectively. That is, $m_{i}^{\rho}\left(B_{i}^{\rho}\right)=\xi_{i}^{\rho}$ and $m_{i}^{\rho}\left(\Theta_{i}^{\rho}\right)=$ $1-\xi_{i}^{\rho}, i=1,2$; for instance, according to public information and relevant knowledge, $B_{1}^{\rho}\left(B_{2}^{\rho}\right), \Theta_{1}^{\rho}\left(\Theta_{2}^{\rho}\right)$, and $\xi_{1}^{\rho}\left(\xi_{2}^{\rho}\right)$ are given by row 2 , row 3 , and row 4 in Table 2 (Table 3), respectively;

(v) $\Omega=\left\{\Omega_{1}, \Omega_{2}\right\}$ is the optimistic attitude set, where $\Omega_{1}=\left\{\omega_{1}^{\rho}\right\}$ and $\Omega_{2}=\left\{\omega_{2}^{\rho}\right\}$ are given by row 6 in Tables 2 and 3 , respectively;

(vi) $U=\left\{u_{1}, u_{2}\right\}$ is the payoff function set, where $u_{1}\left(c_{i}\right)=$ $i-6$ and $u_{2}\left(c_{i}\right)=6-i$, where $i=1, \ldots, 11$; clearly, $u_{1}$ does reflect the defender's preference ordering $c_{11}>_{1} c_{10}>_{1} \cdots>_{1} c_{1}$, and $u_{2}$ reflects the attacker's preference ordering $\left.c_{1}\right\rangle_{2} c_{2} \succ_{2} \cdots \succ_{2} c_{11}$.

Given the above setting, $\beta$-values can be calculated as follows. Since $B_{1}^{\left(a_{1}, a_{1}\right)}=\left\{c_{10}, c_{11}\right\}, B_{1}^{\left(a_{1}, a_{2}\right)}=\left\{c_{5}\right\}, \Theta_{1}^{\left(a_{1}, a_{1}\right)}=$ $\left\{c_{7}, \ldots, c_{11}\right\}$, and $\Theta_{1}^{\left(a_{1}, a_{2}\right)}=\left\{c_{4}, \ldots, c_{7}\right\}$, we have

$$
\begin{gathered}
t_{1}^{\left(a_{1}, a_{1}\right)}=u_{1}\left(c_{10}\right)=10-6=4, \\
T_{1}^{\left(a_{1}, a_{1}\right)}=u_{1}\left(c_{11}\right)=11-6=5 ; \\
t_{1}^{\left(a_{1}, a_{2}\right)}=T_{1}^{\left(a_{1}, a_{2}\right)}=u_{1}\left(c_{5}\right)=5-6=-1 ; \\
y_{1}^{\left(a_{1}, a_{1}\right)}=\min \left\{u_{1}\left(c_{7}\right), \ldots, u_{1}\left(c_{11}\right)\right\}=u_{1}\left(c_{7}\right)=1, \\
Y_{1}^{\left(a_{1}, a_{1}\right)}=\max \left\{u_{1}\left(c_{7}\right), \ldots, u_{1}\left(c_{11}\right)\right\}=u_{1}\left(c_{11}\right)=5 ; \\
y_{1}^{\left(a_{1}, a_{2}\right)}=\min \left\{u_{1}\left(c_{4}\right), \ldots, u_{1}\left(c_{7}\right)\right\}=u_{1}\left(c_{4}\right)=-2, \\
Y_{1}^{\left(a_{1}, a_{2}\right)}=\max \left\{u_{1}\left(c_{4}\right), \ldots, u_{1}\left(c_{7}\right)\right\}=u_{1}\left(c_{7}\right)=1 .
\end{gathered}
$$

TABLE 3: Important elements of the ambiguous game for the attacker.

\begin{tabular}{llcccc}
\hline & $B_{2}^{\rho}$ & $\Theta_{2}^{\rho}$ & $\xi_{2}^{\rho}$ & $\beta_{2}^{\rho}$ & $\omega_{2}^{\rho}$ \\
\hline$\rho=\left(a_{1}, a_{1}\right)$ & $\left\{c_{9}\right\}$ & $\left\{c_{7}, \ldots, c_{11}\right\}$ & 0.8 & $1 / 2$ & $1 / 2$ \\
$\rho=\left(a_{1}, a_{2}\right)$ & $\left\{c_{5}\right\}$ & $\left\{c_{3}, \ldots, c_{6}\right\}$ & 0.4 & $1 / 3$ & $2 / 3$ \\
$\rho=\left(a_{1}, a_{3}\right)$ & $\left\{c_{3}\right\}$ & $\left\{c_{1}, \ldots, c_{5}\right\}$ & 0.7 & $1 / 2$ & $4 / 5$ \\
$\rho=\left(a_{2}, a_{1}\right)$ & $\left\{c_{1}\right\}$ & $\left\{c_{1}, \ldots, c_{5}\right\}$ & 0.6 & 1 & $1 / 3$ \\
$\rho=\left(a_{2}, a_{2}\right)$ & $\left\{c_{7}\right\}$ & $\left\{c_{5}, \ldots, c_{8}\right\}$ & 0.5 & $1 / 3$ & $2 / 3$ \\
$\rho=\left(a_{2}, a_{3}\right)$ & $\left\{c_{3}\right\}$ & $\left\{c_{1}, \ldots, c_{5}\right\}$ & 0.7 & $1 / 2$ & $4 / 5$ \\
$\rho=\left(a_{3}, a_{1}\right)$ & $\left\{c_{1}\right\}$ & $\left\{c_{1}, \ldots, c_{5}\right\}$ & 0.4 & 1 & $1 / 2$ \\
$\rho=\left(a_{3}, a_{2}\right)$ & $\left\{c_{5}\right\}$ & $\left\{c_{3}, \ldots, c_{6}\right\}$ & 0.5 & $1 / 3$ & $1 / 3$ \\
$\rho=\left(a_{3}, a_{3}\right)$ & $\left\{c_{9}\right\}$ & $\left\{c_{7}, \ldots, c_{11}\right\}$ & 0.8 & $1 / 2$ & $2 / 3$ \\
\hline
\end{tabular}

Thus, by formula (14), we have

$$
\begin{aligned}
\beta_{1}^{\left(a_{1}, a_{1}\right)} & =\frac{t_{1}^{\left(a_{1}, a_{1}\right)}-y_{1}^{\left(a_{1}, a_{1}\right)}}{\left(Y_{1}^{\left(a_{1}, a_{1}\right)}-T_{1}^{\left(a_{1}, a_{1}\right)}\right)+\left(t_{1}^{\left(a_{1}, a_{1}\right)}-y_{1}^{\left(a_{1}, a_{1}\right)}\right)} \\
& =\frac{4-1}{5-5+4-1}=1, \\
\beta_{1}^{\left(a_{1}, a_{2}\right)} & =\frac{t_{1}^{\left(a_{1}, a_{2}\right)}-y_{1}^{\left(a_{1}, a_{2}\right)}}{\left(Y_{1}^{\left(a_{1}, a_{2}\right)}-T_{1}^{\left(a_{1}, a_{2}\right)}\right)+\left(t_{1}^{\left(a_{1}, a_{2}\right)}-y_{1}^{\left(a_{1}, a_{2}\right)}\right)} \\
& =\frac{-1+2}{1+2+1-1}=\frac{1}{3} .
\end{aligned}
$$

Similarly, we can calculate the other $\beta$-values for both players, which are given in row 5 of Tables 2 and 3 .

4.3. Ambiguous Equilibrium. To find the ambiguous equilibria of this game, let us first compute the expected utility intervals of the pure strategy profile. Take the pure strategy profile $\rho=\left(a_{1}, a_{1}\right)$ as an example. By formulas (7), we can calculate the lower and upper bound of the expected payoff interval for $\left(a_{1}, a_{1}\right)$ as

$$
\begin{aligned}
\underline{U}_{1}^{\left(a_{1}, a_{1}\right)} & =\xi_{1}^{\left(a_{1}, a_{1}\right)} t_{1}^{\left(a_{1}, a_{1}\right)}+\left(1-\xi_{1}^{\left(a_{1}, a_{1}\right)}\right) y_{1}^{\left(a_{1}, a_{1}\right)} \\
& =0.7 \times u_{1}\left(c_{10}\right)+(1-0.7) u_{1}\left(c_{7}\right) \\
& =0.7 \times 4+0.3 \times 1=3.1, \\
\bar{U}_{1}^{\left(a_{1}, a_{1}\right)} & =\xi_{1}^{\left(a_{1}, a_{1}\right)} T_{1}^{\left(a_{1}, a_{1}\right)}+\left(1-\xi_{1}^{\left(a_{1}, a_{1}\right)}\right) Y_{1}^{\left(a_{1}, a_{1}\right)} \\
& =0.7 \times u_{1}\left(c_{11}\right)+(1-0.7) u_{1}\left(c_{11}\right) \\
& =0.7 \times 5+0.3 \times 5=5 .
\end{aligned}
$$

In this ambiguous game $G$, we associate the pure strategy profile $\left(a_{1}, a_{1}\right)$ with the expected payoff interval $[3.1,5.0]$ for player 1. Similarly, we can obtain the expected payoff intervals associated with the other pure strategy profiles, which are all shown in Table 4. 
TABLE 4: Expected payoff intervals for the ambiguous game $G$.

\begin{tabular}{cccc}
\hline & $a_{1}$ & $a_{2}$ & $a_{3}$ \\
\hline \multirow{2}{*}{$a_{1}$} & {$[3.1,5.0]$,} & {$[-1.6,0.2]$,} & {$[-3.4,-2.6]$,} \\
& {$[-3.4,-2.6]$} & {$[0.4,2.2]$} & {$[2.4,3.6]$} \\
$a_{2}$ & {$[-5.0,-1.9]$,} & {$[1.0,2.5]$,} & {$[-3.8,-2.6]$,} \\
& {$[3.4,5.0]$} & {$[-1.5,0.0]$} & {$[2.4,3.6]$} \\
$a_{3}$ & {$[-5.0,-3.4]$,} & {$[-1.3,-0.7]$,} & {$[2.6,3.4]$,} \\
& {$[2.6,5.0]$} & {$[0.5,2.0]$} & {$[-3.4,-2.6]$} \\
\hline
\end{tabular}

TABLE 5: Payoff matrix of the induced game $G_{1}^{\prime}$.

\begin{tabular}{cccc}
\hline & $a_{1}$ & $a_{2}$ & $a_{3}$ \\
\hline$a_{1}$ & $3.733,-3.0$ & $-0.7,1.6$ & $-3.0,3.36$ \\
$a_{2}$ & $-2.52,3.933$ & $1.75,-0.5$ & $-2.84,3.36$ \\
$a_{3}$ & $-3.933,3.8$ & $-1.0,1.0$ & $3.0,-2.867$ \\
\hline
\end{tabular}

By formula (6), we thus obtain the payoff matrix of induced game $G_{1}^{\prime}=\left(N, S, U^{\prime}\right)$ (as shown in Table 5).

In this ambiguous game, a mixed strategy $\left(p_{1}, p_{2}, p_{3}\right)$ (where $p_{3}=1-p_{1}-p_{2}$ ) can be interpreted as the defender or attacker allocating proportion $p_{i}$ of his available resources to prevent or attack airport $a_{i}(i=1,2,3)$. Let $\alpha=\left(\left(p_{1}, p_{2}, 1-\right.\right.$ $\left.\left.p_{1}-p_{2}\right),\left(q_{1}, q_{2}, 1-q_{1}-q_{2}\right)\right)$ be a mixed-strategy profile. Given the induced game $\left(N, S, U^{\prime}\right)$, we have

$$
\begin{aligned}
E_{1}(\alpha)= & 3.733 p_{1} q_{1}-0.7 p_{1} q_{2}-3 p_{1}\left(1-q_{1}-q_{2}\right) \\
& -2.52 p_{2} q_{1}+1.75 p_{2} q_{2}-2.84 p_{2}\left(1-q_{1}-q_{2}\right) \\
& -3.933\left(1-p_{1}-p_{2}\right) q_{1}-\left(1-p_{1}-p_{2}\right) q_{2} \\
& +3\left(1-p_{1}-p_{2}\right)\left(1-q_{1}-q_{2}\right), \\
E_{2}(\alpha)= & -3 p_{1} q_{1}+1.6 p_{1} q_{2}+3.36 p_{1}\left(1-q_{1}-q_{2}\right) \\
& +3.933 p_{2} q_{1}-0.5 p_{2} q_{2}+3.36 p_{2}\left(1-q_{1}-q_{2}\right) \\
& +3.8\left(1-p_{1}-p_{2}\right) q_{1}+\left(1-p_{1}-p_{2}\right) q_{2} \\
& -2.867\left(1-p_{1}-p_{2}\right)\left(1-q_{1}-q_{2}\right) .
\end{aligned}
$$

Let $\partial E_{1} / \partial p_{i}=0$ and $\partial E_{2} / \partial q_{i}=0(i=1,2)$. Then, we have $p_{1}=0.4211, p_{2}=0.1938, q_{1}=0.2057$, and $q_{2}=0.5062$. One can easily verify that the strategy profile

$$
\alpha^{*}=((0.4211,0.1938,0.3851),(0.2057,0.5062,0.2881))
$$

is the unique mixed-strategy equilibrium of induced game $G^{\prime}$. According to Theorem $8, \alpha^{*}$ is also the only ambiguous equilibrium of the ambiguous game $G$. Given this equilibrium point, it follows from formulas $(25)$ that $\Xi_{1}\left(\alpha^{*}\right)=-0.4508$ and $\Xi_{2}\left(\alpha^{*}\right)=0.9620$.

4.4. Relation between Focal Mass Values and Solutions. This subsection illustrates the relationship between the focal mass values and the solutions of an ambiguous game, and how to manage private information under such a situation.

In the above analysis, each focused mass $\xi_{1}^{\rho}$ is given as a constant. Now, assume that all the focused masses are
TABLE 6: Payoff matrix of the induced game $G_{2}^{\prime}$.

\begin{tabular}{cccc}
\hline & $a_{1}$ & $a_{2}$ & $a_{3}$ \\
\hline$a_{1}$ & $4.333,-3.00$ & $-0.50,2.00$ & $-3.00,4.20$ \\
$a_{2}$ & $-1.80,5.00$ & $2.00,0.00$ & $-2.60,4.20$ \\
$a_{3}$ & $-2.333,5.00$ & $-1.25,1.00$ & $3.00,-3.667$ \\
\hline
\end{tabular}

variables. Then, according to Theorem 9 and values $\beta_{1}^{\rho}$ in row 5 and values $\omega_{1}^{\rho}$ in row 6 of Table 2 , for the defender and any mixed-strategy profile $\alpha$, we have the following.

(i) Since $\omega_{1}^{\left(a_{1}, a_{1}\right)}<\beta_{1}^{\left(a_{1}, a_{1}\right)}$ and $\omega_{1}^{\left(a_{2}, a_{2}\right)}<\beta_{1}^{\left(a_{2}, a_{2}\right)}$, the function $\Xi_{1}\left(\xi_{1}^{\left(a_{1}, a_{1}\right)}, \ldots, \xi_{1}^{\left(a_{3}, a_{3}\right)}\right)$ is strictly increasing in the coordinates $\xi_{1}^{\left(a_{1}, a_{1}\right)}$ and $\xi_{1}^{\left(a_{2}, a_{2}\right)}$. This means that the more the information represented by $m_{1}^{\left(a_{1}, a_{1}\right)}$, $\left(m_{1}^{\left(a_{2}, a_{2}\right)}\right)$ the better for the defender.

(ii) Since $\omega_{1}^{\left(a_{1}, a_{2}\right)}>\beta_{1}^{\left(a_{1}, a_{2}\right)}, \omega_{1}^{\left(a_{2}, a_{1}\right)}>\beta_{1}^{\left(a_{2}, a_{1}\right)}, \omega_{1}^{\left(a_{2}, a_{3}\right)}>$ $\beta_{1}^{\left(a_{2}, a_{3}\right)}$, and $\omega_{1}^{\left(a_{3}, a_{1}\right)}>\beta_{1}^{\left(a_{3}, a_{1}\right)}$, the function $\Xi_{1}\left(\xi_{1}^{\left(a_{1}, a_{1}\right)}\right.$, $\left.\ldots, \xi_{1}^{\left(a_{3}, a_{3}\right)}\right)$ is strictly decreasing in the coordinates $\xi_{1}^{\left(a_{1}, a_{2}\right)}, \xi_{1}^{\left(a_{2}, a_{1}\right)}, \xi_{1}^{\left(a_{2}, a_{3}\right)}$, and $\xi_{1}^{\left(a_{3}, a_{1}\right)}$. This means that the less information represented by $m_{1}^{\left(a_{1}, a_{2}\right)}\left(m_{1}^{\left(a_{2}, a_{1}\right)}\right.$, $m_{1}^{\left(a_{2}, a_{3}\right)}$, and $\left.m_{1}^{\left(a_{3}, a_{1}\right)}\right)$ the better for the defender.

(iii) Since $\omega_{1}^{\left(a_{1}, a_{3}\right)}=\beta_{1}^{\left(a_{1}, a_{3}\right)}, \omega_{1}^{\left(a_{3}, a_{2}\right)}=\beta_{1}^{\left(a_{3}, a_{2}\right)}$, and $\omega_{1}^{\left(a_{3}, a_{3}\right)}=\beta_{1}^{\left(a_{3}, a_{3}\right)}$, the function $\Xi_{1}\left(\xi_{1}^{\left(a_{1}, a_{1}\right)}, \ldots, \xi_{1}^{\left(a_{3}, a_{3}\right)}\right)$ is independent of the coordinates $\xi_{1}^{\left(a_{1}, a_{3}\right)}, \xi_{1}^{\left(a_{3}, a_{2}\right)}$, and $\xi_{1}^{\left(a_{3}, a_{3}\right)}$

(iv) The function $\Xi_{1}\left(\xi_{1}^{\left(a_{1}, a_{1}\right)}, \ldots, \xi_{1}^{\left(a_{3}, a_{3}\right)}\right)$ has its maximum value at point $(1,0, x, 0,1,0,0, y, z)$, where $x, y, z \in$ $[0,1]$.

Similarly, for the attacker and any mixed-strategy profile $\alpha$, the function $\Xi_{2}\left(\xi_{2}^{\left(a_{1}, a_{1}\right)}, \ldots, \xi_{2}^{\left(a_{3}, a_{3}\right)}\right)$ achieves its maximum value at point $\left(x^{\prime}, 0,0,1,0,0,1, y^{\prime}, 0\right)$, where $x^{\prime}, y^{\prime} \in[0,1]$.

Given these critical points, we can compute the lower and upper expected payoffs for each pure strategy profile using formulas (7). Thus, we obtain by formula (6) the payoff matrix of the induced game $G_{2}^{\prime}=\left(N, S, U^{\prime \prime}\right)$ as shown in Table 6 . It is easy to show that

$$
\alpha^{\star}=((0.4631,0.1679,0.3690),(0.2164,0.4828,0.3008))
$$

is the unique mixed-strategy equilibrium of induced game $G_{2}^{\prime}$. Thus, by Theorem $8, \alpha^{\star}$ is the unique ambiguous mixedstrategy equilibrium of the ambiguous game $G$. Given this equilibrium point, we can obtain $\Xi_{1}\left(\alpha^{\star}\right)=-0.2060$ and $\Xi_{2}\left(\alpha^{\star}\right)=1.295$, which are the maximums of the defender's and attacker's expected payoffs given by the equilibria of the ambiguous game. Indeed, we have that $\Xi_{1}\left(\alpha^{\star}\right)>\Xi_{1}\left(\alpha^{*}\right)$ and $\Xi_{2}\left(\alpha^{\star}\right)>\Xi_{2}\left(\alpha^{*}\right)$.

In order to provide a geometric illustration, let us consider a special case that is helpful in demonstrating the relation between the mass value and the ambiguous equilibrium. Let $\xi_{1}^{\left(a_{1}, a_{1}\right)}$ and $\xi_{1}^{\left(a_{1}, a_{2}\right)}$ be two variables, and let the 


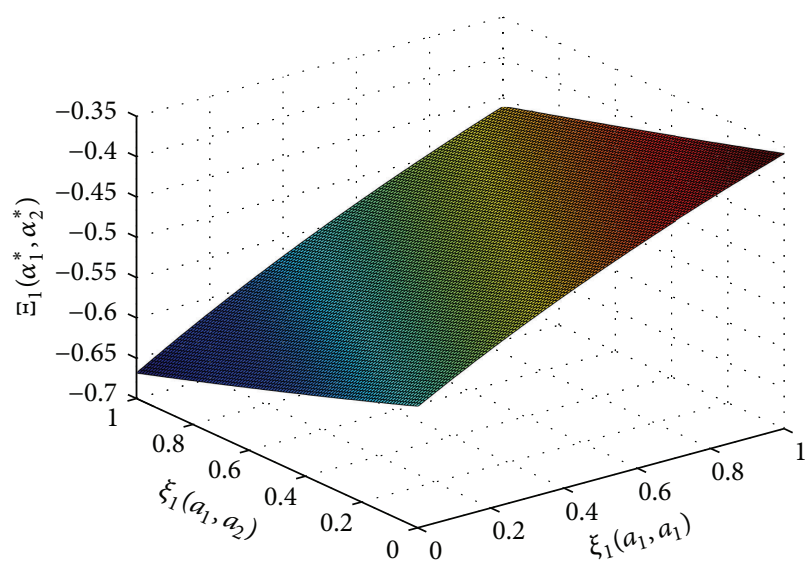

FIGURE 1: Generalized expected payoffs at the ambiguous equilibria.

other focused masses be constants as those shown in Table 2 . Since $\beta_{1}^{\left(a_{1}, a_{1}\right)}>\omega_{1}^{\left(a_{1}, a_{1}\right)}$ and $\beta_{1}^{\left(a_{1}, a_{2}\right)}<\omega_{1}^{\left(a_{1}, a_{2}\right)}$, by item (iv) of Theorem 9, the function $\Xi_{1}$ achieves its maximum value at the critical point $(1,0)$. In view of this, we obtain that the mixed-strategy profile

$$
\alpha^{*}=((0.4211,0.1938,0.3851),(0.1801,0.5278,0.2921))
$$

is the ambiguous equilibrium with maximum value $\Xi_{1}\left(\alpha^{*}\right)=$ -0.3598 . Figure 1 depicts the relation between the focal mass values $\xi_{1}^{\left(a_{1}, a_{1}\right)}, \xi_{1}^{\left(a_{1}, a_{2}\right)}$, and $\Xi_{1}\left(\alpha^{*}\right)$. One can observe from the figure that the function $\Xi_{1}\left(\alpha^{*}\right)$ is strictly increasing in the coordinate $\xi_{1}^{\left(a_{1}, a_{1}\right)}$ and is strictly decreasing in the coordinate $\xi_{1}^{\left(a_{1}, a_{2}\right)}$

\section{Discussion}

In the literature, the Bayesian game [11] is widely used to model interactive situations under ambiguity. Basically, the framework of Bayesian games assumes that a player's belief regarding the other players' types is accurate. In other words, it is assumed that the player has a precise probability distribution over the set of the types. In particular, the player should assign an equal probability to each type in the case of complete ignorance. In this framework, the expected payoff of each strategy profile can be computed, and thus a preference ordering can be determined. However, many researches $[2,3,24]$ have shown that the belief of a decision maker may not be representable by a precise probability distribution when ambiguity occurs. In contrast, our model employs Dempster-Shafer theory, an instance of theories of imprecise probability, to model ambiguity and thus relaxes the fundamental assumption of Bayesian games.

The Choquet expected utility theory is a natural extension of the expected utility theory to decision making under ambiguity. To deal with ambiguity in games, some papers [1418] apply the Choquet expected utility theory to the context of games, which leads to a Choquet expected payoff for each strategy profile. In these approaches, the players maximize the Choquet expected payoffs, from which a preference ordering can be determined. It is worth pointing out that these approaches assume the consequence of a strategy profile to be determinate. By contrast, our model relaxes such an assumption. Another problem with these approaches is that their solutions, called equilibria in games, may not exist in some cases [29]. However, we have established that there is at least one ambiguous equilibrium in any ambiguous game.

Based on the Choquet expected utility theory, Marinacci [17] studies the ambiguous games by considering pessimism and optimism towards ambiguity, which are two different kinds of extreme attitudes. Unlike his framework, our model resolves ambiguity using the Hurwicz criterion, which is a weighted average of the purely optimistic and purely pessimistic value according to the degree of optimism. As shown in Corollary 10, each player's outcomes will become worse if he/she is too optimistic or pessimistic. In order to model ambiguity in games, researchers $[30,31]$ use the model of multiple priors (a set of probabilities) to represent players' beliefs and employ the maximin decision rule to determine a preference ordering. Such a decision rule requires the decision maker to choose an option that maximizes the minimal expected utilities with respect to the set of multiple priors. Based on these ideas, they provide some analysis for games where players' beliefs are modeled by multiple priors. As a matter of fact, the Hurwicz criterion is much more general in comparison with the maximin decision rule. Therefore, our game model based on the Hurwicz criterion enables us to investigate how different kinds of attitudes towards ambiguity may affect the solutions of an ambiguous game.

\section{Conclusion}

In real life, we often need to make decisions under uncertainty. In particular, we may not have sufficient evidence to determine a precise probability distribution over the set of possible consequences of a choice. In this paper, we have provided a new decision-theoretic framework for decision making under uncertainty by applying Dempster-Shafer theory to model ambiguity and the Hurwicz criterion to determine a preference ordering. Based on this decision-theoretic framework, we have developed a game-theoretic model for analyzing games where players may be ambiguous about consequences of some strategy profile. In order to determine the outcomes of such games, we have proposed a new solution concept called ambiguous equilibrium. We have also studied in this paper how players' optimistic attitudes and their beliefs about the consequences may influence the possible solutions of an ambiguous game. Moreover, we have shown that overly optimistic or pessimistic attitude would make players' expected payoff worse in ambiguous games.

There are some interesting projects that deserve further investigation. One natural future project is to study the consequences of applying our theory to extensive form games and cooperative games. One can consider Dempster's rule of combination as the rule for updating based on new information in ambiguous games. It also seems natural to investigate the consistency of the optimistic attitudes that different players have towards the same strategy profile. Finally, another important 
project for future work is to find some simple algorithm for solving an ambiguous game.

\section{Conflict of Interests}

The author declares that there is no conflict of interests regarding the publication of this paper.

\section{Acknowledgments}

The author would like to thank Matthias Troffaes, Hailin Liu, Xudong Luo, Gert de Cooman, and Teddy Seidenfeld for their helpful comments on earlier versions of this paper. The author is also extremely grateful to Guiomar Martin-Herran and three referees for their insightful comments, which have significantly improved the paper. This paper is supported by National Fund of Philosophy and Social Science from China (no. 11BZX060), and major projects of National Fund of Philosophy and Social Science from China (no. 13 \& ZD186).

\section{References}

[1] D. Ellsberg, "Risk, ambiguous, and the savage axioms," Quarterly Journal of Economics, vol. 75, no. 4, pp. 643-669, 1961.

[2] I. Levi, "Why indeterminate probability is rational," Journal of Applied Logic, vol. 7, no. 4, pp. 364-376, 2009.

[3] A. Snow, "Amgibuity and the value of information," Journal of Risk and Uncertainty, vol. 40, no. 2, pp. 133-145, 2010.

[4] T.-Y. Chen, "Optimistic and pessimistic decision making with dissonance reduction using interval-valued fuzzy sets," Information Sciences, vol. 181, no. 3, pp. 479-502, 2011.

[5] A. I. Houston, P. C. Trimmer, T. W. Fawcett, A. D. Higginson, J. A. R. Marshall, and J. M. McNamara, "Is optimism optimal? Functional causes of apparent behavioural biases," Behavioural Processes, vol. 89, no. 2, pp. 172-178, 2012.

[6] W. Xiong and H. Liu, "On solving some paradoxes using the ordered weighted averaging operator based decision model," International Journal of Intelligent Systems, vol. 29, no. 1, pp. 125, 2014.

[7] W. Xiong and H. Liu, "An axiomatic foundationfor Yager's decision theory," International Journal of Intelligent Systems. In press.

[8] M. Clemente, F. R. Fernandez, and J. Puerto, "Pareto-optimal security strategies in matrix games with fuzzy payoffs," Fuzzy Sets and Systems, vol. 176, no. 1, pp. 36-45, 2011.

[9] W. Ma, W. Xiong, and X. Luo, "A model for decision making with missing, imprecise, and uncertain evalutions of multiple criteria," International Journal of Intelligent Systems, vol. 28, no. 2, pp. 152-184, 2013.

[10] J. Xiong, X. Tan, K. Yang, and Y. Chen, "Fuzzy group decision making for multiobjective problems: tradeo between consensus and robustness," Journal of Applied Mathematics, vol. 2013, Article ID 657978, 9 pages, 2013.

[11] J. C. Harsanyi, "Games with incomplete information played by "Bayesian" players: I-III-part I: The Basic Model," Management Science, vol. 14, no. 3, pp. 159-182, 1967.

[12] M. Larbani, "Solving bimatrix games with fuzzy payoffs by introducing nature as a third player," Fuzzy Sets and Systems, vol. 160, no. 5, pp. 657-666, 2009.
[13] L. Mallozzi, V. Scalzo, and S. Tijs, "Fuzzy interval cooperative games," Fuzzy Sets and Systems, vol. 165, no. 1, pp. 98-105, 2011.

[14] S. Bade, "Ambiguous act equilibria," Games and Economic Behavior, vol. 71, no. 2, pp. 246-260, 2011.

[15] J. Eichberger and D. Kelsey, "Are the treasures of game theory ambiguous?" Economic Theory, vol. 48, no. 2-3, pp. 313-339, 2011.

[16] R. Kozhan, "Non-additive anonymous games," International Journal of Game Theory, vol. 40, no. 2, pp. 215-230, 2011.

[17] M. Marinacci, "Ambiguous games," Games and Economic Behavior, vol. 31, no. 2, pp. 191-219, 2000.

[18] G. de Marco and M. Romaniello, "Beliefs correspondences and equilibria in ambiguous games," International Journal of Intelligent Systems, vol. 27, no. 2, pp. 86-107, 2012.

[19] D. Schmeidler, "Subjective probability and expected utility without additivity," Econometrica, vol. 57, no. 3, pp. 571-587, 1989.

[20] W. Xiong, X. Luo, and W. Ma, "Games with ambiguous payoffs and played by ambiguity and regret minimising players," in Proceedings of the 25th Anniversary of the Australian Joint Conference on Artifficial Intelligence, pp. 409-420, 2012.

[21] K. J. Arrow and L. Hurwicz, "An optimality criterion for decision-making under ignorance," in Uncertainty and Expectations in Economics Essays in Honour of G.L.S. Shackle, Basil Blackwell, Oxford, UK, 1972.

[22] R. Guillanume and P. Zielinski, "Decision making under scenario uncertainty in a requirement planning," in Proceedings of the 14th International Conference on Information Processing and Management of Uncertainty in Knowledge-Based Systems, pp. 104-113, 2012.

[23] G. Jeantet and O. Spanjaard, "Optimizing the Hurwicz criterion in decision trees with imprecise probabilities," in Proceedings of the 1st International Conference on Algorithmic Decision Theory, pp. 340-352, 2009.

[24] G. Shafer, A Mathematical Theory of Evidence, Princeton University Press, Princeton, NJ, USA, 1976.

[25] T. M. Strat, "Decision analysis using belief functions," International Journal of Approximate Reasoning, vol. 4, no. 5-6, pp. 391418, 1990.

[26] G. Shafer and A. Tversky, "Languages and designs for probability judgment," Cognitive Science, vol. 9, no. 3, pp. 309-339, 1985.

[27] H. Gintis, The Bounds of Reason: Game Theory and the Unification of the Behavioral Sciences, Princeton University Press, Princeton, NJ, USA, 2009.

[28] J. Nash, "Non-cooperative games," Annals of Mathematics, vol. 54, no. 2, pp. 286-295, 1951.

[29] K. Ritzberger, "On games under expected utility with rank dependent probabilities," Theory and Decision, vol. 40, no. 1, pp. 1-27, 1996.

[30] A. Kajii and T. Ui, "Incomplete information games with multiple priors," Japanese Economic Review, vol. 56, no. 3, pp. 332-351, 2005.

[31] K. C. Lo, "Equilibrium in beliefs under uncertainty," Journal of Economic Theory, vol. 71, no. 2, pp. 443-484, 1996. 


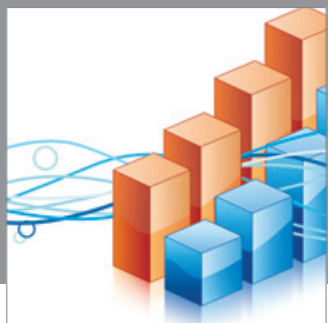

Advances in

Operations Research

mansans

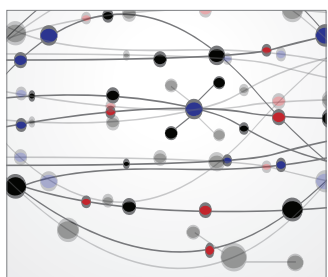

The Scientific World Journal
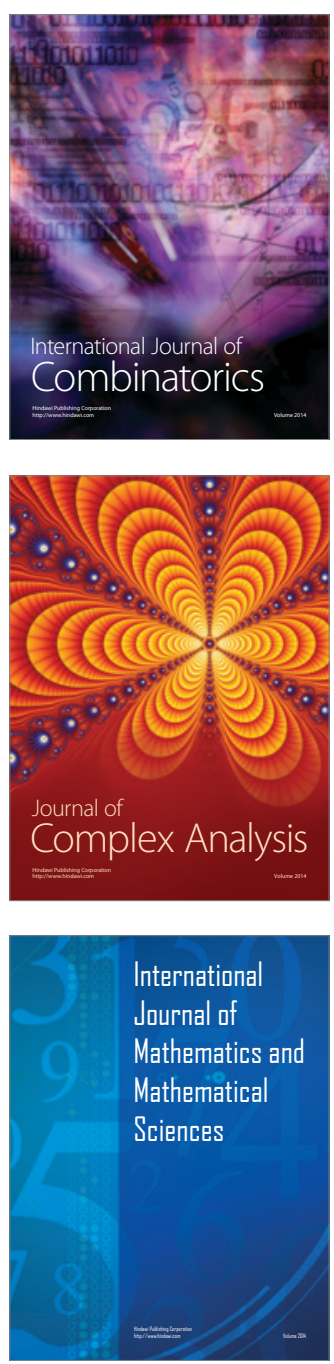
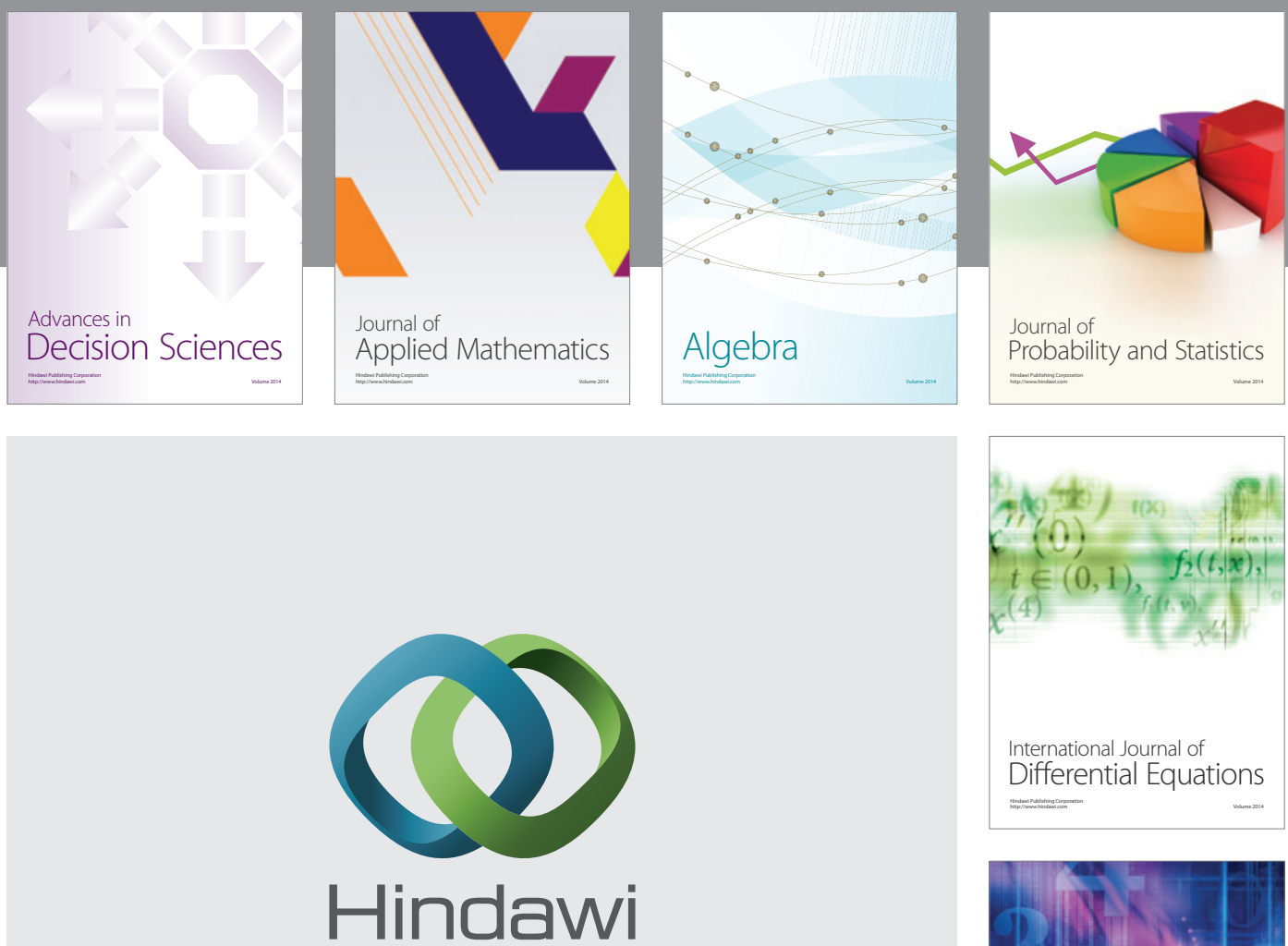

Submit your manuscripts at http://www.hindawi.com
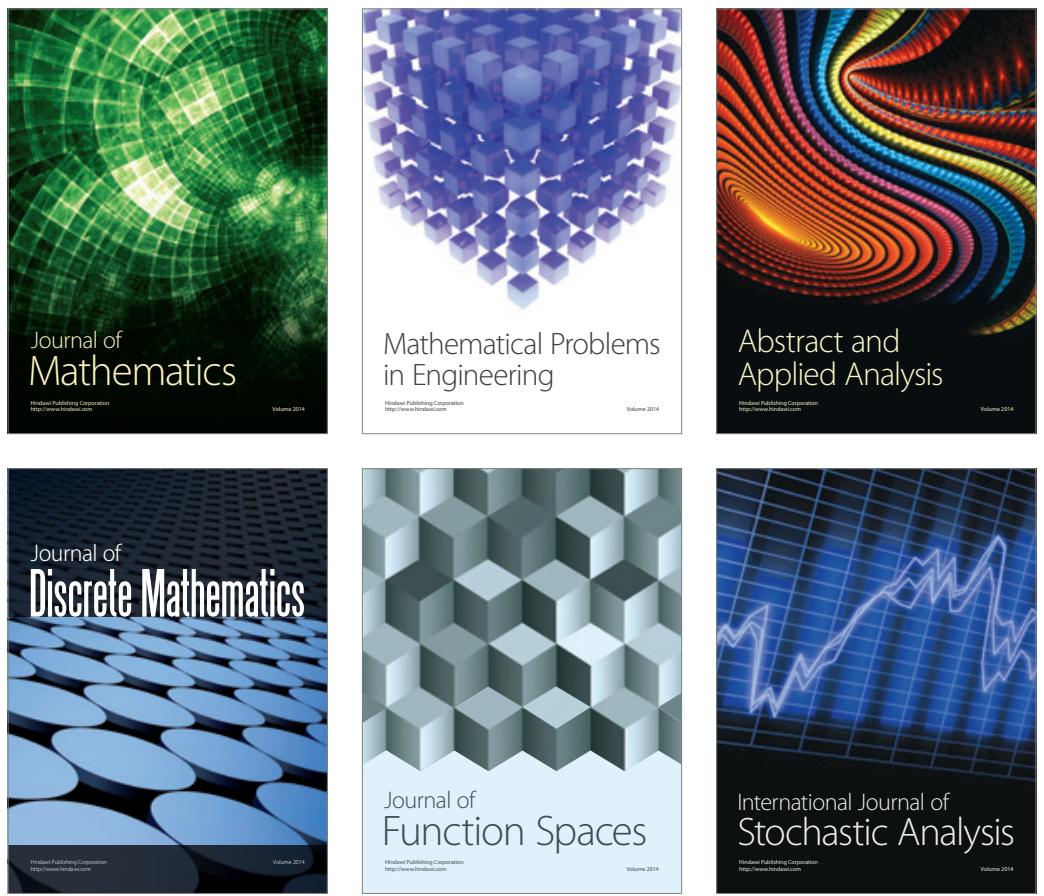

Journal of

Function Spaces

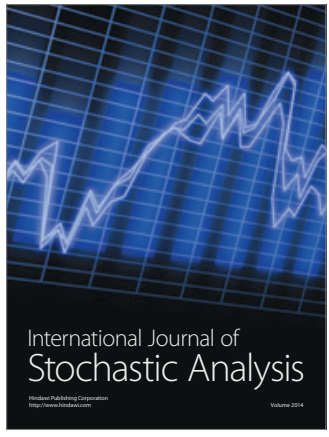

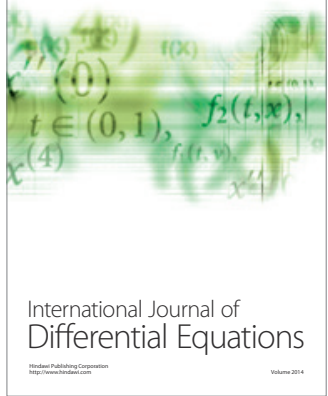
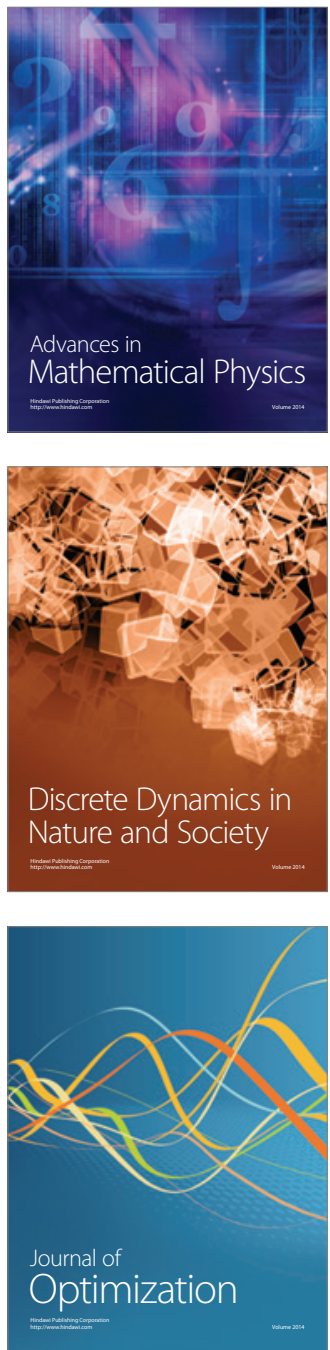